PROCEEDINGS OF THE

AMERICAN MATHEMATICAL SOCIETY

Volume 134, Number 4, Pages 1051-1057

S 0002-9939(05)08092-5

Article electronically published on July 21, 2005

\title{
EIGENVALUES OF SCALING OPERATORS AND A CHARACTERIZATION OF $B$-SPLINES
}

\author{
XIAOJIE GAO, S. L. LEE, AND QIYU SUN
}

(Communicated by David R. Larson)

\begin{abstract}
A finitely supported sequence $a$ that sums to 2 defines a scaling operator $T_{a} f=\sum_{k \in \mathbb{Z}} a(k) f(2 \cdot-k)$ on functions $f$, a transition operator $S_{a} v=$ $\sum_{k \in \mathbb{Z}} a(k)(2 \cdot-k)$ on sequences $v$, and a unique compactly supported scaling function $\phi$ that satisfies $\phi=T_{a} \phi$ normalized with $\widehat{\phi}(0)=1$. It is shown that the eigenvalues of $T_{a}$ on the space of compactly supported square-integrable functions are a subset of the nonzero eigenvalues of the transition operator $S_{a}$ on the space of finitely supported sequences, and that the two sets of eigenvalues are equal if and only if the corresponding scaling function $\phi$ is a uniform $B$-spline.
\end{abstract}

\section{INTRODUCTION}

A finitely supported real sequence $a:=\{a(k)\}_{k \in \mathbb{Z}}$, normalized so that $\sum_{k \in \mathbb{Z}} a(k)$ $=2$, defines the scaling operator $T_{a}$ on $L^{p}(\mathbb{R}), 1 \leq p \leq \infty$, by

$$
T_{a} f:=\sum_{k \in \mathbb{Z}} a(k) f(2 \cdot-k), \quad f \in L^{p}(\mathbb{R}),
$$

and the transition operator $S_{a}$ on $\ell^{p}(\mathbb{Z})$ by

$$
\left(S_{a} v\right)(j):=\sum_{k \in \mathbb{Z}} a(k) v(2 j-k), \quad v \in \ell^{p}(\mathbb{Z}) .
$$

We shall deal mainly with the space $L_{c}^{2} \equiv L_{c}^{2}(\mathbb{R})$ of compactly supported $L^{2}$ functions, and $\ell_{0} \equiv \ell_{0}(\mathbb{Z})$ the space of finitely supported sequences.

In the Fourier transform domain (1.1) becomes

$$
\widehat{T_{a} f}(u)=H(u / 2) \widehat{f}(u / 2)
$$

where $H(u)=\frac{1}{2} \sum_{k \in \mathbb{Z}} a(k) e^{-i k u}$. The infinite product $\prod_{n=1}^{\infty} H\left(2^{-n} u\right)$ converges locally uniformly, and there exists a compactly supported distribution $\phi$ whose Fourier transform is

$$
\widehat{\phi}(u)=\prod_{n=1}^{\infty} H\left(2^{-n} u\right),
$$

Received by the editors October 25, 2004.

2000 Mathematics Subject Classification. Primary 41A15, 41A99, 42C40, 65T60.

Key words and phrases. Scaling operators, transition operators, eigenvalues, uniform $B$ splines. 
which satisfies

$$
\widehat{\phi}(u)=H(u / 2) \widehat{\phi}(u / 2)
$$

or equivalently

$$
T_{a} \phi=\phi \text { and } \widehat{\phi}(0)=1 .
$$

The compactly supported distribution $\phi$ is indeed the unique solution of (1.5) (see [4, [5). The equation (1.5) is known as a scaling equation or refinement equation. Its solution is called a scaling function, and the sequence $a$ is called its mask.

Scaling functions play an important role in multiscale representation, which has applications in scale-space analysis ([18]), geometric modelling ([3], 9]) and wavelet analysis (4, [5]). Many properties of a scaling function are controlled by the spectrum of its transition operator $([5,6,17,11,12,14,17])$. For a nice account of properties of transition operators and their adjoints, the subdivision operators, see $([1,2,8,19,20)$. In this paper we study the relationship between the spectra of the scaling operator and the transition operator defined by a sequence $a$. Let $\sigma_{e}(T, X)$ denote the set of eigenvalues of a continuous linear operator $T$ on a Banach space $X$. The object is to show that the inclusion $\sigma_{e}\left(T_{a}, L_{c}^{2}\right) \cup\{0\} \subset \sigma_{e}\left(S_{a}, \ell_{0}\right)$ holds for any $a \in \ell_{0}$, with equality if and only if the mask $a$ is a shift of a binomial sequence, i.e. $\widehat{a}(u)=2 \cdot e^{-i L u}\left(\frac{1+e^{-i u}}{2}\right)^{k}$, and hence the corresponding refinable function is a $B$-spline. In Section 2 we prove that the inclusion $\sigma_{e}\left(T_{a}, L_{c}^{2}\right) \cup\{0\} \subset \sigma_{e}\left(S_{a}, \ell_{0}\right)$ holds for all $a \in \ell_{0}$, and in Section 3 it is shown that equality holds if and only if the mask $a$ is a shift of the binomial sequence. In fact in Theorem 3.2 we prove more and the results are more precise.

\section{EigenVAlues OF SCALING AND TRANSition OPERATORS}

In this section we develop a relationship between the eigenvalues of the scaling operator $T_{a}$ on $L_{c}^{2}$ and the corresponding transition operator $S_{a}$ on $\ell_{0}$. We shall establish the following theorem.

Theorem 2.1. Let $a:=\{a(k)\}_{k \in \mathbb{Z}} \in \ell_{0}$ satisfy $\sum_{k \in \mathbb{Z}} a(k)=2$. Then

$$
\sigma_{e}\left(T_{a}, L_{c}^{2}\right) \cup\{0\} \subset \sigma_{e}\left(S_{a}, \ell_{0}\right) .
$$

As a consequence of Theorem 2.1 we have the following result $([3,6])$.

Corollary 2.2. Let $a:=\{a(k)\}_{k \in \mathbb{Z}} \in \ell_{0}$ satisfy $\sum_{k \in \mathbb{Z}} a(k)=2$. Then

$$
\sigma_{e}\left(T_{a}, C_{c}(\mathbb{R})\right) \cup\{0\} \subset \sigma_{e}\left(S_{a}, \ell_{0}\right),
$$

where $C_{c}(\mathbb{R})$ is the space of all compactly supported continuous functions on $\mathbb{R}$.

We shall first establish two lemmas in the run up to the proof of Theorem 2.1.

Lemma 2.3. Let $a:=\{a(k)\}_{k \in \mathbb{Z}} \in \ell_{0}$ satisfy $\sum_{k \in \mathbb{Z}} a(k)=2$. Then $\sigma_{e}\left(T_{a}, L_{c}^{2}\right)=\emptyset$ or $\left\{1,1 / 2, \ldots, 1 / 2^{k_{0}}\right\}$ for some nonnegative integer $k_{0}$. In the later situation, the unique compactly supported solution $\phi$ of the refinement equation $T_{a} \phi=\phi$ with $\widehat{\phi}(0)=1$ has derivatives up to order $k_{0}$ in $L^{2}(\mathbb{R})$ and if $g_{l}$ is an eigenfunction of the scaling operator $T_{a}$ with eigenvalue $2^{-l}, l=0,1, \ldots, k_{0}$, then $g_{l}=C \phi^{(l)}$ for some nonzero constant $C$. 
Proof. Let $\lambda \in \sigma_{e}\left(T_{a}, L_{c}^{2}\right)$ and $g_{\lambda}$ be a nonzero function in $L_{c}^{2}$ that satisfies

$$
T_{a} g_{\lambda}=\lambda g_{\lambda} \text {. }
$$

Then it suffices to prove that $\lambda=2^{-l}$ and $g_{\lambda}=C \phi^{(l)}$ for some nonnegative integer $l$. Taking the Fourier transform at both sides of (2.3) leads to

$$
H(u / 2) \widehat{g}_{\lambda}(u / 2)=\lambda \widehat{g}_{\lambda}(u),
$$

where $H(u):=\frac{1}{2} \sum_{k \in \mathbb{Z}} a(k) e^{-i k u}$. Note that $\widehat{g}_{\lambda}$ is analytic on $\mathbb{R}$ since $g_{\lambda}$ has compact support. Therefore, by comparing the order of $u$ on both sides of equation (2.4) at the origin and using $H(0)=1$, we conclude that $\lambda=2^{-l}$ for some nonnegative integer $l$. Thus $G_{\lambda}(u):=\widehat{g}_{\lambda}(u) u^{-l}$ is still analytic and satisfies the equation $G_{\lambda}(u)=H(u / 2) G_{\lambda}(u / 2)$. This shows that $G_{\lambda}(u)=G_{\lambda}(0) \widehat{\phi}(u)$ since $\widehat{\phi}(u)=\prod_{n=1}^{\infty} H\left(2^{-n} u\right)$. Hence $g_{\lambda}=C \phi^{(l)}$ for some nonzero constant $C$.

The next lemma is a known result on a sum rule of an $L^{2}$-function, which we shall state here for convenient reference (see, for instance, [10] and the references therein).

Lemma 2.4. For $a=\{a(k)\}_{k \in \mathbb{Z}} \in \ell_{0}$ that satisfies $\sum_{k \in \mathbb{Z}} a(k)=2$, let $\phi$ be the unique compactly supported distributional solution of the refinement equation $\phi=T_{a} \phi$. If $\phi, \ldots, \phi^{\left(k_{0}\right)} \in L^{2}(\mathbb{R})$, then the symbol $H(u):=\frac{1}{2} \sum_{k \in \mathbb{Z}} a(k) e^{-i k u}$ satisfies

$$
H(u)=\left(\frac{1+e^{-i u}}{2}\right)^{k_{0}+1} \widetilde{H}(u)
$$

for some trigonometric polynomial $\widetilde{H}(u)$.

Proof of Theorem 2.1. The assertion is trivial if $\sigma_{e}\left(T_{a}, L_{c}^{2}\right)=\emptyset$ since one can easily see that $\{0\} \subset \sigma_{e}\left(S_{a}, \ell_{0}\right)$. So we assume that $\sigma_{e}\left(T_{a}, L_{c}^{2}\right) \neq \emptyset$. By Lemma 2.3.

$$
\sigma_{e}\left(T_{a}, L_{c}^{2}\right)=\left\{1, \ldots, 2^{-k_{0}}\right\}
$$

for some nonnegative integer $k_{0}$, and $\phi, \phi^{\prime}, \ldots, \phi^{\left(k_{0}\right)} \in L_{c}^{2}$. Thus $\phi, \phi^{\prime}, \ldots, \phi^{\left(k_{0}-1\right)}$ are compactly supported continuous functions. Taking derivatives on both sides of the equation $T_{a} \phi=\phi$, gives

$$
\phi^{(l)}=2^{l} T_{a} \phi^{(l)}, \quad l=0,1, \ldots, k_{0}-1 .
$$

Let $S$ be the sampling operator on the integers, i.e. $S: C(\mathbb{R}) \rightarrow \ell(\mathbb{Z})$ such that $S f:=\{f(k)\}_{k \in \mathbb{Z}} \forall f \in C(\mathbb{R})$. Applying the sampling operator $S$ and using the commutation identity $S T_{a}=S_{a} S$, leads to

$$
S_{a} S \phi^{(l)}=2^{-l} S \phi^{(l)}, \quad l=0,1, \ldots, k_{0}-1 .
$$

We claim that $S \phi^{(l)}$ is not a zero sequence. Suppose on the contrary that $S \phi^{(l)} \equiv$ 0 , i.e. $\phi^{(l)}(k)=0$ for all $k \in \mathbb{Z}$. Using (2.6) inductively on $n \in \mathbb{Z}_{+}$, we have $\phi^{(l)}\left(2^{-n} k\right)=0$ for all $k \in \mathbb{Z}$, which together with the continuity of $\phi^{(l)}$ leads to $\phi^{(l)} \equiv 0$. Hence $\phi$ is a polynomial, which contradicts the fact that $\phi$ is a nonzero compactly supported function. This proves our claim that $S \phi^{(l)}$ is not a zero sequence. Hence $2^{-l}, l=0,1, \ldots, k_{0}-1$, are eigenvalues of the operator $S_{a}$ on $\ell_{0}$ by (2.7) and the above claim. Therefore, it remains to prove that $2^{-k_{0}} \in \sigma_{e}\left(S_{a}, \ell_{0}\right)$. Recall that $\phi, \phi^{\prime}, \ldots, \phi^{\left(k_{0}\right)} \in L_{c}^{2}$ by Lemma 2.3. Then

$$
H(u)=\left(\frac{1+e^{-i u}}{2}\right)^{k_{0}+1} \widetilde{H}(u),
$$


for some trigonometric polynomial $\widetilde{H}(u)$ by Lemma 2.4, Setting

$$
H_{1}(u)=\left(\frac{1+e^{-i u}}{2}\right) \widetilde{H}(u)
$$

and writing $H_{1}(u)=\sum_{k \in \mathbb{Z}} a_{1}(k) e^{-i k u}$, we have

$$
\sum_{k \in \mathbb{Z}} a_{1}(2 k)=\sum_{k \in \mathbb{Z}} a_{1}(2 k+1)=1 .
$$

Let $N_{1}$ be so chosen that $a_{1}(k)=0$ for all integer $k$ with $|k|>N_{1}$. Then by (2.9), the sum of the entries of every column of the matrix $B:=\left(a_{1}(2 i-j)\right)_{i, j=-N_{1}}^{N_{1}}$ is 1 . Thus 1 is an eigenvalue of $B$. Hence there exists a nonzero vector $v_{1}=$ $\left(v_{1}\left(-N_{1}\right), \ldots, v_{1}\left(N_{1}\right)\right)^{T}$ such that $B v_{1}=v_{1}$. Considered as a compactly supported sequence, $v_{1}$ has Fourier series $\widehat{v}_{1}(u)=\sum_{k=-N_{1}}^{N_{1}} v_{1}(k) e^{-i k u}$. Then

$$
\begin{aligned}
\widehat{v}_{1}(2 u) & =\left(B v_{1}\right)^{\wedge}(2 u)=\sum_{j, k=-N_{1}}^{N_{1}} a_{1}(2 j-k) v_{1}(k) e^{-2 i j u} \\
& =\sum_{j, k \in \mathbb{Z}} a_{1}(2 j-k) v_{1}(k) e^{-2 i j u}=\left(S_{a_{1}} v_{1}\right)^{\wedge}(2 u) .
\end{aligned}
$$

By (2.8) and (2.10), the nonzero finitely supported sequence $w \in \ell_{0}$ defined by $\widehat{w}(u)=\left(1-e^{-i u}\right)^{k_{0}} \widehat{v}_{1}(u)$ satisfies

$$
\begin{aligned}
\left(S_{a} w\right)^{\wedge}(u) & =H(u) \widehat{w}(u / 2)+H(u / 2+\pi) \widehat{w}(u / 2+\pi) \\
& =2^{-k_{0}}\left(1-e^{-i u}\right)^{k_{0}}\left(H_{1}(u / 2) \widehat{v}_{1}(u / 2)+H_{1}(u / 2+\pi) \widehat{v}_{1}(u / 2+\pi)\right) \\
& =2^{-k_{0}}\left(1-e^{-i u}\right)^{k_{0}}\left(S_{a_{1}} v_{1}\right)^{\wedge}(u) \\
& =2^{-k_{0}}\left(1-e^{-i u}\right)^{k_{0}} \widehat{v}_{1}(u)=2^{-k_{0}} \widehat{w}(u) .
\end{aligned}
$$

This proves that $2^{-k_{0}} \in \sigma_{e}\left(S_{a}, \ell_{0}\right)$, and hence completes the proof of Theorem 2.1 .

\section{SCALING OPERATORS AND $B$-SPLINES}

For a sequence $a:=\{a(k)\}_{k \in \mathbb{Z}} \in \ell_{0}$ let $I_{a} \subset \mathbb{Z}$ be the smallest interval containing the support of $a$. For an interval $I \subset \mathbb{Z}$ let $\ell(I):=\left\{a \in \ell_{0}: \operatorname{supp}(a) \subset I\right\}$. One may verify that $\ell\left(I_{a}\right)$ is an invariant subspace of $S_{a}$ on $\ell_{0}$, i.e. $S_{a} v \in \ell\left(I_{a}\right)$ for all $v \in \ell\left(I_{a}\right)$. For an integer $N \geq 1$, let $a_{N}=\left\{a_{N}(k)\right\}_{k \in \mathbb{Z}}$ be the binomial sequence of order $N$, i.e. $a_{N}(k)=\frac{1}{2^{N-1}}\left(\begin{array}{c}N \\ k\end{array}\right)$ for $k=0,1, \ldots, N$, and 0 otherwise. The corresponding scaling operator $T_{a_{N}}$ and the transition operator $S_{a_{N}}$ restricted to compactly supported $L^{2}$-functions and sequences supported in $I_{a_{N}}$ respectively have the same eigenvalues as described in the following theorem.

Theorem 3.1. If $a_{N}$ is the binomial sequence of order $N$, then

$$
\begin{gathered}
\sigma_{e}\left(T_{a_{N}}, L_{c}^{2}\right)=\left\{2^{-k}: k=0,1, \ldots, N-1\right\}, \\
\sigma_{e}\left(S_{a_{N}}, \ell\left(I_{a_{N}}\right)\right)=\left\{2^{-k}: k=0,1, \ldots, N-1\right\},
\end{gathered}
$$

and

$$
\sigma_{e}\left(S_{a_{N}}, \ell_{0}\right)=\{0\} \cup\left\{2^{-k}: k=0,1, \ldots, N-1\right\} .
$$


For a sequence $a:=\{a(k)\}_{k \in \mathbb{Z}} \in \ell_{0}$ that satisfies $\sum_{k \in \mathbb{Z}} a(k)=2$, Theorem 2.1 says that $\sigma_{e}\left(T_{a}, L_{c}^{2}\right) \cup\{0\} \subset \sigma_{e}\left(S_{a}, \ell_{0}\right)$. On the other hand, Theorem 3.1 shows that equality is attained for the binomial sequences $a_{N}$ for any $N \in \mathbb{N}$. A natural question is whether equality holds for any other sequences $a \in \ell_{0}$ besides the binomial sequences. The answer is no and the next result shows why not.

Theorem 3.2. Let $a:=\{a(k)\}_{k \in \mathbb{Z}} \in \ell_{0}$ satisfy $\sum_{k \in \mathbb{Z}} a(k)=2$. Then the following are equivalent:

(a) The mask a is a shifted binomial sequence, i.e.

$$
\frac{1}{2} \sum_{k \in \mathbb{Z}} a(k) e^{-i k u}=e^{-i L u}\left(\frac{1+e^{-i u}}{2}\right)^{N}=2^{-N} e^{-i L u} \sum_{k=0}^{N}\left(\begin{array}{c}
N \\
k
\end{array}\right) e^{-i k u}
$$

where $L=\max \left(I_{a}\right)$ and $N=\left|I_{a}\right|$.

(b) $\sigma_{e}\left(S_{a}, \ell\left(I_{a}\right)\right)=\sigma_{e}\left(T_{a}, L_{c}^{2}\right)$.

(c) $\sigma_{e}\left(S_{a}, \ell_{0}\right)=\sigma_{e}\left(T_{a}, L_{c}^{2}\right) \cup\{0\}$ and $0 \notin \sigma_{e}\left(S_{a}, \ell\left(I_{a}\right)\right)$.

Refinement equations whose masks are shifted binomial sequences arise in many situations. The corresponding refinable functions are $B$-splines, which have many desirable properties ideal for signal processing [16] and geometric modelling [3]. Here the $N$-th order $B$-spline $B_{N}$ is defined inductively by $B_{N+1}:=B_{N} * B_{1}$ with $B_{1}:=\chi_{[0,1]}$. Theorem 3.2 characterizes $B$-splines via the connection between the eigenvalues of the corresponding scaling operators and transition operators on spaces of compactly supported $L^{2}$-functions and finitely supported sequences respectively. See ([13, 15]) and references therein for more characterizations of $B$-splines.

To prove Theorem 3.1, we need a lemma.

Lemma 3.3. Let $a:=\{a(k)\}_{k \in \mathbb{Z}} \in \ell_{0}$ satisfy $\sum_{k \in \mathbb{Z}} a(k)=2$. Then

$$
\sigma_{e}\left(S_{a}, \ell\left(I_{a}\right)\right) \cup\{0\}=\sigma_{e}\left(S_{a}, \ell\left(I_{a} \cup\left(I_{a}+1\right)\right)\right)=\sigma_{e}\left(S_{a}, \ell_{0}\right) .
$$

Proof. From the matrix representation of $S_{a}$, it follows that

$$
0 \in \sigma_{e}\left(S_{a}, \ell\left(I_{a} \cup\left(I_{a}+1\right)\right)\right) .
$$

Because of (3.5), the following inclusions hold:

$$
\sigma_{e}\left(S_{a}, \ell\left(I_{a}\right)\right) \cup\{0\} \subset \sigma_{e}\left(S_{a}, \ell\left(I_{a} \cup\left(I_{a}+1\right)\right)\right) \subset \sigma_{e}\left(S_{a}, \ell_{0}\right) .
$$

Then we need only to show that $\sigma_{e}\left(S_{a}, \ell_{0}\right) \subset \sigma_{e}\left(S_{a}, \ell\left(I_{a}\right)\right) \cup\{0\}$. Note that $I_{\left(S_{a} v\right)}=$ $\frac{I_{a}+I_{v}}{2} \cap \mathbb{Z}$ for any $v \in \ell_{0}$. Then if $\lambda \in \sigma_{e}\left(S_{a}, \ell_{0}\right) \backslash\{0\}$ and $0 \neq v_{\lambda} \in \ell_{0}$ satisfies $S_{a} v_{\lambda}=\lambda v_{\lambda}$, then $I_{v_{\lambda}} \subset I_{a}$, which means that $v_{\lambda} \in \ell\left(I_{a}\right)$ and $\lambda \in \sigma_{e}\left(S_{a}, \ell\left(I_{a}\right)\right)$.

Proof of Theorem 3.1. We first prove (3.1). Since the $B$-spline $B_{N}$ is a piecewise polynomial of degree $N-1$ of compact support and $B_{N} \in C^{N-2}$ for $N \geq 1$, it follows that $B_{N}^{(n)} \in L_{c}^{2}$ for any $n=0,1, \ldots, N-1$, and $B_{N}^{(N)} \notin L_{c}^{2}$. Therefore, the first assertion (3.1) follows from Lemma 2.3

Next we prove (3.2). For the binomial sequence $a_{N}$, we have $I_{a_{N}}=[0, N] \cap \mathbb{Z}$ and $\widehat{a}_{N}(u)=2^{-N+1}\left(1+e^{-i u}\right)^{N}$. One may verify that the functions $F_{k}, 1 \leq k \in \mathbb{Z}$, defined by $F_{1}(u):=1$ and

$$
F_{k}(u):=\sum_{j \in \mathbb{Z}}\left(\frac{e^{-i(u+2 j \pi)}-1}{-i(u+2 j \pi)}\right)^{k}=\sum_{j \in \mathbb{Z}} B_{k}(j) e^{-i j u},
$$


satisfy

$$
F_{k}(u)=\left(\frac{1+e^{-i u / 2}}{2}\right)^{k} F_{k}\left(\frac{u}{2}\right)+\left(\frac{1+e^{-i(u / 2+\pi)}}{2}\right)^{k} F_{k}\left(\frac{u}{2}+\pi\right), k \geq 1 .
$$

Therefore by the proof of (2.11), for any $k=0, \ldots, N-1$, the sequence $v_{k}$ with $\widehat{v}_{k}(u)=\left(1-e^{-i u}\right)^{k} F_{N-k}(u), k=0,1, \ldots, N-1$, belong to $\ell\left(I_{a_{N}}\right)$ and are the eigensequences of the transition operator $S_{a_{N}}$ associated with the eigenvalue $2^{-k}$. This yields

$$
\left\{2^{-k}: k=0,1, \ldots, N-1\right\} \subset \sigma_{e}\left(S_{a_{N}}, \ell\left(I_{a_{N}}\right)\right) .
$$

One may also verify that the sequences $v$, whose Fourier transforms are $\left(1-e^{-i u}\right)^{N-1}$ and $\left(1-e^{-i u}\right)^{N-1} e^{-i u}$, belong to $\ell\left(I_{a_{N}}\right)$ and are linearly independent eigenvectors of the transition operator $S_{a_{N}}$ associated with the eigenvalue $2^{-N+1}$. By (3.6) and the above fact, there are $N$ distinct eigenvalues for the operator $S_{a_{N}}$ on $\ell\left(I_{a_{N}}\right)$ and the eigenspace associated with the eigenvalue $2^{-N+1}$ is at least two. Recall that the dimension of the space $\ell\left(I_{a_{N}}\right)$ is $N+1$. Then

$$
\sigma_{e}\left(S_{a_{N}}, \ell\left(I_{a_{N}}\right)\right) \subset\left\{2^{-k}: k=0,1, \ldots, N-1\right\} .
$$

Hence (3.2) follows from (3.6) and (3.7).

Finally, the assertion (3.3) follows easily from (3.2) and Lemma 3.3 .

Proof of Theorem 3.2. For any $l \in \mathbb{Z}, \tau_{l}: \ell_{0} \rightarrow \ell_{0}$ denotes the shift operator $\tau_{l} a=$ $a(\cdot+l)$. We remark that $\sigma_{e}\left(S_{a}, \ell_{0}\right)=\sigma_{e}\left(S_{\tau_{l} a}, \ell_{0}\right), \sigma_{e}\left(S_{a}, \ell\left(I_{a}\right)\right)=\sigma_{e}\left(S_{\tau_{l} a}, \ell\left(I_{\tau_{l} a}\right)\right)$, and $\sigma_{e}\left(T_{a}, L_{c}^{2}\right)=\sigma_{e}\left(T_{\tau_{l} a}, L_{c}^{2}\right)$. So we can assume without loss of generality that $I_{a}=\{0, \ldots, N\}$ for some positive integer $N$.

That (a) implies (b) is the result of Theorem 3.1, while the equivalence between (c) and (b) follows from (2.2) and (3.4) and the fact that $0 \notin \sigma_{e}\left(T_{a}, L_{c}^{2}\right)$. Now we show that (b) implies (a). By Lemma 2.3, $\sigma_{e}\left(T_{a}, L_{c}^{2}\right)=\emptyset$ or $\left\{1,1 / 2, \ldots, 1 / 2^{k_{0}}\right\}$ for some nonnegative integer $k_{0}$. Since $a\left(\min \left(I_{a}\right)\right), a\left(\max \left(I_{a}\right)\right) \in \sigma_{e}\left(S_{a}, \ell\left(I_{a}\right)\right)$ by the matrix representation of $S_{a}$, it follows that

$$
\sigma_{e}\left(S_{a}, \ell\left(I_{a}\right)\right)=\left\{1,1 / 2, \ldots, 1 / 2^{k_{0}}\right\} .
$$

Since as an operator on $\ell\left(I_{a}\right), S_{a}$ is represented by the matrix $(a(2 i-j))_{i, j=0}^{N}$, its eigenpolynomial is of the form $(-1)^{N+1} \lambda^{N+1}+(-1)^{N} \operatorname{trace}\left(S_{a}\right) \lambda^{N}+g(\lambda)$, where $\operatorname{trace}\left(S_{a}\right)=\sum_{j=0}^{N} a(j)=2$ and $g$ is a polynomial of degree less than $N$. Thus the sum of all the eigenvalues of $S_{a}$, counting multiplicity is equal to $\operatorname{trace}\left(S_{a}\right)=2$. Therefore, assuming that $1 / 2^{k}$ is an eigenvalue of $S_{a}$ on $\ell\left(I_{a}\right)$ with multiplicity $l_{k} \geq 1$, we obtain the equations $\sum_{k=0}^{k_{0}} l_{k} / 2^{k}=2$ and $\sum_{k=0}^{k_{0}} l_{k}=N+1$. The first equation yields $l_{k}=1$ for $k=0,1, \ldots, k_{0}-1, l_{k_{0}}=2$. The second equation then implies

$$
k_{0}=N-1 .
$$

By (3.8), (3.9) and assumption (b), we obtain $\sigma_{e}\left(T_{a}, L_{c}^{2}\right)=\left\{1,1 / 2, \cdots, 1 / 2^{N-1}\right\}$. This together with Lemmas 2.3 and 2.4 and the fact $I_{a}=\{0, \ldots, N\}$ prove that $H(u):=\frac{1}{2} \sum_{k \in \mathbb{Z}} a(k) e^{-i k u}=\left(\frac{1+e^{-i u}}{2}\right)^{N}$, and hence assertion (a). 


\section{REFERENCES}

1. O. Bratteli and P. Jorgensen, Wavelets through a looking glass, Birkhäuser Boston, Inc., Boston, MA, 2002. MR1913212 (2003i:42001)

2. C. A. Cabrelli, S. B. Heineken and U. M. Molter, Local Bases for refinable spaces, preprint.

3. A. S. Cavaretta, W. Dahmen and C. A. Micchelli, Stationary subdivision, Memoir Amer. Math. Soc., 93(1991), 1-186. MR1079033 (92h:65017)

4. C. K. Chui, An Introduction to Wavelets, Academic Press, New York, 1992. MR:1150048 (93f:42055)

5. I. Daubechies, Ten Lectures on Wavelets, CBMS-NSF Regional Conference Series in Applied Mathematics, SIAM, Philadelphia, 1992. MR1162107 (93e:42045)

6. I. Daubechies and J. Lagarias, Two-scale difference equation I: existence and global regularity of solutions, SIAM J. Math. Anal., 22(1991), 1388-1410. MR1112515 (92d:39001)

7. T. Eirola, Sobolev characterization of solutions of dilation equation, SIAM J. Math. Anal., 23(1992), 1015-1030. MR1166573 (93f:42056)

8. X. Gao, S. L. Lee and Q. Sun, Spectrum of Transition, Subdivision and Multiscale operators, Wavelet Analysis (Hong Kong 2001) (edited by D. Zhou), World Science Publishing, 2002, 123-138. MR1941608 (2003j:42034)

9. R. Q. Jia, Subdivision schemes in $L^{p}$ spaces, Adv. Comput. Math., 3(1995), 309-341. MR1339166 (96d:65028)

10. R. Q. Jia, Approximation properties of multivariate wavelets, Math. Comp., 67(1998), 647665. MR1451324 (98g:41020)

11. R. Q. Jia, Characterization of smoothness of multivariate refinable functions in Sobolev spaces, Trans. Amer. Math. Soc., 351(1999), 4089-4112. MR.1473444 (99m:42050)

12. W. Lawton, Necessary and sufficient conditions for constructing orthonormal wavelet bases, J Math. Physics, 32(1991), 57-61. MR1083085 (91m:81100)

13. W. Lawton, S. L. Lee and Z. Shen, Characterization of compactly supported refinable splines, Adv. Comput. Math., 3(1995), 137-145. MR1314906 (95m:41020)

14. A. Ron and Z. Shen, The Sobolev regularity of refinable functions, J. Approx. Theory, 106(2000), 185-225. MR 1788272 (2001j:42034)

15. Q. Sun and Z. Zhang, A characterization of compactly supported both $m$ and $n$ refinable distributions, J. Approx. Theory, 99(1999), 198-216. MR1696549 (2001e:42051)

16. M. Unser, Splines: a perfect fit for signal and image processing, IEEE Signal and Image Proc. Magazine, 16(1999), no. 6., 22-38.

17. L. F. Villemoes, Wavelet analysis of refinement equations, SIAM J. Math. Anal., 25(1994), 1433-1460. MR1289147 (96f:39009)

18. Y. P. Wang and S. L. Lee, Scale-space derived from B-spline, IEEE Trans. on Pattern Analysis and Machine Intelligence, 20(1998), 1040-1055.

19. D. Zhou, Spectra of subdivision operators, Proc. Amer. Math. Soc. 129(2001), no. 1, 191-202. MR $1784023(2001 \mathrm{~h}: 47049)$

20. D. Zhou, Two-scale homogeneous functions in wavelet analysis, J. Fourier Anal. Appl., 8(2002), no. 6, 565-580. MR1932746 (2003i:42060)

Department of Mathematics, National University of Singapore, 2 Science Drive 2, SingAPORE 117543

E-mail address: matgxj@nus.edu.sg

Department of Mathematics, National University of Singapore, 2 Science Drive 2, SiNGAPORE 117543

E-mail address: matleesl@nus.edu.sg

Department of Mathematics, National University of Singapore, 2 Science Drive 2, SINGAPORE 117543

E-mail address: matsungy@nus.edu.sg 